\title{
Normative Measurement of Orbital Structures in Computed Tomography
}

\author{
Damodar Rokka1, Sharma Poudel ${ }^{2}$, Prakash Kayastha², Sundar Suwal', Sudil Poudyal ${ }^{3}$, Saroj \\ Chhetry ${ }^{4}$, Benu Lohani ${ }^{2}$, Shantalall Shrestha ${ }^{2}$, Rakesh Karn ${ }^{5}$ \\ 'Departement of Radiology, National Trauma Center, National Academy of Medical Sciences (NAMS), Kathmandu, \\ 2Department of Radiology, Maharajgunj Medical Campus, Tribhuvan University Teaching Hospital, Institute of \\ Medicine, Kathmandu, ${ }^{3}$ Department of Radiology, Chitwan Medical College, Chitwan. ${ }^{4}$ Department of Radiology, \\ Sahid Gangalal National Heart Center, Kathmandu, ${ }^{5}$ Department of Radiology, Grande International Hospital, \\ Kathmandu
}

\section{Corresponding author:}

Prakash Kayastha, MBBS, MD

Department of Radiology, Maharajgunj Medical Campus, Tribhuvan University Teaching Hospital, Institute of Medicine, Kathmandu

Email: dr_prakash_kayastha@hotmail.com

Submitted : Oct 4, 2020

Accepted : Nov 29, 2020

\begin{abstract}
\section{Introduction}

Normal orbital dimensions are important for diagnosing orbital pathologies. Computed tomography (CT) of the orbit is an important modality offering high accuracy for precise measurements of orbit. The study was disbursed to ascertain criteria for the normative values of the various orbital dimensions using computed tomography in patients visiting Tribhuvan University Teaching Hospital, Kathmandu.
\end{abstract}

\section{Methods}

Orbital volume, ocular diameter, length of the interzygomatic line (IZL), the distance of the posterior extent of the globe from the interzygomatic line, globe position, diameters of extraocular muscles (medial, lateral, superior group, and inferior rectus) and lens density were measured in each eye of 172 patients undergoing $\mathrm{CT}$ examination of paranasal sinuses. The correlations with age and sex were also analyzed.

\section{Results}

Mean values (mean $\pm \mathrm{SD}$ ) of orbital volume, ocular diameter, IZL, globe position and lens density were $24.54 \pm 2.57 \mathrm{~cm}^{3}, 2.44 \pm 0.22 \mathrm{~cm}, 94.3 \pm 6.2 \mathrm{~mm}, 12.3 \pm 2.4 \mathrm{~mm}$ and $141.43 \pm 16.62 \mathrm{HU}$ respectively. Mean diameters of extraocular muscles were $3.67 \pm 0.52 \mathrm{~mm}, 3.36 \pm 0.50 \mathrm{~mm}, 3.74 \pm 0.42 \mathrm{~mm}$ and $3.87 \pm 0.38 \mathrm{~mm}$ for medial rectus, lateral rectus, inferior rectus and the superior group respectively. There was no significant age and gender difference in most of the measurements. There was significant positive correlation of age with lens density, orbital volume and ocular diameter $(p<0.05)$.

\section{Conclusion}

Normative data of different orbital measurements were obtained. The present result may help radiologists and ophthalmologists to accurately assess various orbital parameters, particularly in Nepalese populations.

\section{Keywords}

$C T$, measurements, orbit 


\section{INTRODUCTION}

$\mathrm{E}$ yes are the sense organs for vision. The configuration of the structures of the eye and their mechanisms are set optimally for vision, such that some diseases that affect the dimensions of the eye or its component may cause visual abnormalities. ${ }^{1}$ The normal orbital dimensions like orbital volume, the diameters of extraocular muscles, the optic nerve-sheath complex, the globe position and computed tomography (CT) attenuation values of lens are very important while diagnosing and differentiating orbital diseases.

Enlargement of the extraocular muscles (EOM) can occur in various pathologies e.g. primary neoplasm, non-specific inflammations, metastatic tumors, vascular malformations etc., as well as Graves' ophthalmopathy, the most common cause. ${ }^{2-4}$ Optic nerve sheath diameter (ONSD) is important because changes in ONSD may indicate changes of an intracranial pressure. Different authors indicated a normal/abnormal threshold (a cutoff value) of the diameter from $4.8 \mathrm{~mm}$ to $5.9 \mathrm{~mm}$ with numerous variations between these values. ${ }^{5,6}$ Change in orbital volume $(\mathrm{OV})$ is a changing process. Short and longterm changes occur due to aging of soft tissue and bony structures resulting changes in shape, size, and volume of the bony orbit. ${ }^{78}$ Measurement of OV can be inconsistent because of the complicated anatomical structures of the bony orbit. CT of the orbit is an important modality offering high accuracy for precise evaluation of such findings.

CT attenuation of the cataractous lens is markedly less than the contralateral lens due to increased fluid within the lens. Traumatized globe can have an acute traumatic cataract or have a cataract developed within a 1-year follow-up period, which will show reduced lens density. ${ }^{9}$

There is relative paucity of literature and data on normal dimensions of orbital structures and OV measurements involving Asians on CT imaging and none (to the best of our knowledge) such study has been done in our population. Therefore, this CT-based study was done to help establish criteria for the normal diameters of healthy extraocular muscles, the optic nerve sheath as well as the diameter of the eyeball, the normal position of the globe in the orbit, orbital volume, the CT attenuation value of normal eye lens and their variations with different age and sex.

\section{METHODS}

This was a prospective cross-sectional study carried out in the Department of Radiology and Imaging, Tribhuvan University Teaching Hospital, Kathmandu from July 2017 to October 2017. The study population consisted of a total of 344 orbits in 172 patients who were referred for $\mathrm{CT}$ examinations of Paranasal
Sinuses (PNS) for various clinical indications. Purposive sampling method was used. Ethical clearance was obtained from Institutional Review Board (IRB) of the Institute of Medicine. Informed written consent was obtained from the subjects before the study. The privacy and confidentiality of the patients enrolled in this study were strictly maintained.

The patients without clinical evidence or history of endocrine diseases (e.g. thyroid disease) or any orbital disorder/ trauma were included in the study. Suboptimal scans with artifacts from dental material, patient motion or eyeball implant that might cause errors in orbit measurements, and CT scans with abnormal orbital findings were excluded.

Volume scan of CT PNS was obtained with MDCT scanner (Siemens Somatom definition AS+) with the standard protocol of the department. Coronal images were reformatted almost perpendicular to the axial plane. Patients were asked to maintain forward gaze and gentle eye closure during the scans to prevent asymmetric extraocular muscle contraction. Because every change in the window settings and slice thickness resulted in different measurements in the same CT scan, all images were reviewed at constant window level/width settings of 50/300 and with the slice thickness of $3 \mathrm{~mm}$ respectively. All the $\mathrm{CT}$ images were formally reported by the radiologists following which the image analysis was carried out only in the patients with normal orbits. The software used was Syngo. via software available in the workstation.

The images were adequately magnified to allow easy placement of the cursors of the measuring calipers. To determine the normal globe position in $\mathrm{CT}$, the interzygomatic line (IZL) at the midglobe section was used as a reference line. The length of the IZL represents the transversal head size, and was defined by the anterior extent of the lateral bony orbital rims. The globe position (GP) was given as the perpendicular distance between the interzygomatic line and the posterior margin of the globe. The optic nerve sheath diameter (ONSD) of both eyes were also measured at $10 \mathrm{~mm}$ distance from the globe, perpendicular to its course in the axial sections. 10

The superior rectus and the levator palpebrae superior muscle were measured together as a single muscle group as superior group (SG) because they could not be reliably distinguished from each other. We measured diameters of the superior group (SG) and inferior rectus (IR) muscles on coronal section and lateral rectus (LR) and medial rectus (MR) muscles were measured on axial CT section.

Ocular diameters (OD) were obtained with axial length (defined as the distance through the visual axis from the anterior corneal surface to the posterior wall of the choroid in axial view 
and it included the anterior chamber depth, lens thickness, and vitreous length) and width (defined as the maximum transverse distance between the temporal and nasal ends of the globe in axial view). The slices chosen for ocular measurements were mid-ocular slices showing the maximum axial size of the eyeball, the lens and optic nerve as well as the insertions of the medial and lateral rectus muscles.

For every patient, orbital volume (OV) was calculated by a 3D-assisted semi-automated volumetric methodology using the $3 \mathrm{D}$ volume rendering tool (VR) available on Syngo.via workstation. The measurement area of the orbit ranged from the orbital septa to the optic canal.

The attenuation of the lens was measured by drawing a region of interest selecting $\mathrm{ROI}$ tool available in the software in the center of eye lens. The average attenuation in Hounsfield Unit (HU) displayed was recorded as the lens density (LD).

Data were collected on an Excel spreadsheet (Microsoft Corp., Redmond, WA, USA). Data were analyzed with Statistical Package for Social Studies (SPSS 23) (IBM Corp., New York, NY, USA). The data were tested for a normal distribution. The mean, standard deviation and range were calculated. The variation of $O V$, diameters of $M R, I R, L R, S G, O D$ and LD by different age groups was analyzed. The independent-sample t-test was used to compare data obtained from male and female patients as well as the right and the left orbit to check statistical significance at the $\mathrm{p}=0.05$ level. The difference of means and 95\% confidence interval (Cl) were used to evaluate the variations by gender and different age groups. Correlation analyses have been performed using the method of Karl-Pearson. Group comparisons were made using parametric and nonparametric two-tailed t-tests wherever appropriate and indicated. A $p$ value $<0.05$ was considered statistically significant.

\section{RESULTS}

Among 172 individuals included in the study, 93 were males $(54.1 \%)$ and 79 females $(45.9 \%)$. Mean age was $34.42 \pm 15.38$ years with range of $11-75$ years.

Mean orbital volume and ocular diameter were $24.54 \mathrm{~cm}^{3}$ and $24.4 \mathrm{~mm}$ respectively. Maximum thickness of extraocular muscles was of superior group $(3.87 \mathrm{~mm})$ and lateral rectus was the thinnest $(3.36 \mathrm{~mm})$. Mean globe position, optic nerve sheath diameter and lens density were $12.3 \mathrm{~mm}, 3.97 \mathrm{~mm}$ and 12.3 HU respectively. (Table 1) There was no statistically significant difference found between data for the right and the left orbits for all the measurements $(p>0.05)$. All of the measurements showed higher values in males than in females in inferior Rectus Diameter as well which showed higher value in females. (Table 1) Statistically, there was no significant difference between male and female subjects in most of the measurements $(p>0.05)$ with the notable exception in the OV and $O D$ which showed the statistically significant difference between males and females $(p<0.05)$.

In relation to age, all of the measurements showed a tendency of increment up to the age of 50 . The largest value of all the measurements were noted in the age group of 41-50 years (Table 2).

There was no statistically significant correlation of age with the size of extraocular muscles diameter, IZL and the position of the globe ( $p>0.05)$. However, a significant weak positive correlation was found between age and density of the eye lens with ' $r$ ' value of $0.19(p<0.05)$. Similarly, a statistically moderate positive correlation was found between age and $\mathrm{OV}$ as well as between age and $\mathrm{OD}$ with the ' $r$ ' values of 0.47 and 0.48 respectively $(p, 0.05)$. Similarly, there was statistically significant but weakly positive correlation of IZL with the size of all the extraocular muscles $(p<0.05)$ and when correlated with the $\mathrm{OV}$, it showed positive correlation with ' $r$ ' value of 0.30 .

Table 1. Measurements of orbital structures in Computed Tomography $(n=344)$

\begin{tabular}{lcccc}
\hline \multicolumn{1}{c}{ Measurements } & $\begin{array}{c}\text { Male } \\
(\text { Mean } \pm \text { SD) }\end{array}$ & $\begin{array}{c}\text { Female } \\
(\text { Mean } \pm \text { SD) }\end{array}$ & $\begin{array}{c}\text { Total } \\
(\text { Mean } \pm \text { SD) }\end{array}$ & Range \\
\hline Orbital volume $\left(\mathrm{cm}^{3}\right)$ & $24.94 \pm 2.52$ & $24.05 \pm 2.53$ & $24.54 \pm 2.57$ & $17.46-29.85$ \\
Ocular diameter $(\mathrm{mm})$ & $24.8 \pm 1.8$ & $23.8 \pm 2.3$ & $24.4 \pm 2.2$ & $18.5-29.0$ \\
Medial rectus $(\mathrm{mm})$ & $3.72 \pm 0.50$ & $3.60 \pm 0.52$ & $3.67 \pm 0.52$ & $2.56-4.85$ \\
Lateral rectus $(\mathrm{mm})$ & $3.39 \pm 0.50$ & $3.30 \pm 0.49$ & $3.36 \pm 0.50$ & $1.88-4.28$ \\
Superior Group $(\mathrm{mm})$ & $3.87 \pm 0.38$ & $3.85 \pm 0.36$ & $3.87 \pm 0.38$ & $2.89-5.16$ \\
Inferior rectus $(\mathrm{mm})$ & $3.72 \pm 0.42$ & $3.74 \pm 0.42$ & $3.74 \pm 0.42$ & $2.80-4.56$ \\
Interzygomatic line $(\mathrm{mm})$ & $94.3 \pm 6.1$ & $94 \pm 6.0$ & $94.3 \pm 6.2$ & $81.5-112.5$ \\
Globe position $(\mathrm{mm})$ & $12.8 \pm 2.4$ & $11.8 \pm 2.2$ & $12.3 \pm 2.4$ & $7.2-18.5$ \\
Optic nerve sheath diameter $(\mathrm{mm})$ & $3.98 \pm 0.54$ & $3.94 \pm 0.48$ & $3.97 \pm 0.51$ & $2.75-5.10$ \\
Lens density (HU) & $140.78 \pm 16.61$ & $141.06 \pm 16.70$ & $141.43 \pm 16.62$ & $112-185$ \\
\hline
\end{tabular}


Table 2. Measurements of orbital structures in Computed Tomography in different age groups $(n=344)$

\begin{tabular}{|c|c|c|c|c|c|c|c|}
\hline \multirow{2}{*}{$\begin{array}{c}\text { Measure- } \\
\text { ments* }\end{array}$} & \multicolumn{7}{|c|}{ Age group } \\
\hline & $11-20$ & $21-30$ & $31-40$ & $41-50$ & $51-60$ & $61-70$ & $71-80$ \\
\hline $\mathrm{OV}\left(\mathrm{cm}^{3}\right)$ & $21.82 \pm 1.88$ & $23.93 \pm 2.44$ & $25.63 \pm 1.95$ & $26.57 \pm 1.76$ & $24.94 \pm 1.84$ & $27.16 \pm 1.99$ & $28.22 \pm 0.16$ \\
\hline $\mathrm{OD}(\mathrm{mm})$ & $22.4 \pm 2.4$ & $24.2 \pm 2.2$ & $24.4 \pm 1.8$ & $24.9 \pm 2.1$ & $25.4 \pm 1.6$ & $25.7 \pm 0.6$ & $26 \pm 2.5$ \\
\hline $\mathrm{MR}(\mathrm{mm})$ & $3.28 \pm 0.41$ & $3.78 \pm 0.39$ & $3.82 \pm 0.45$ & $4.05 \pm 0.32$ & $3.58 \pm 0.52$ & $3.49 \pm 0.41$ & $3.42 \pm 0.24$ \\
\hline $\mathrm{LR}(\mathrm{mm})$ & $2.98 \pm 0.36$ & $3.31 \pm 0.57$ & $3.54 \pm 0.25$ & $3.64 \pm 0.47$ & $3.39 \pm 0.28$ & $3.24 \pm 0.19$ & $3.73 \pm 0.42$ \\
\hline $\mathrm{SG}(\mathrm{mm})$ & $3.72 \pm 0.29$ & $3.89 \pm 0.38$ & $3.98 \pm 0.31$ & $4.08 \pm 0.47$ & $3.68 \pm 0.24$ & $3.5 \pm 0.21$ & $3.48 \pm 0.30$ \\
\hline $\mathrm{IR}(\mathrm{mm})$ & $3.56 \pm 0.36$ & $3.92 \pm 0.36$ & $4.07 \pm 0.27$ & $4.10 \pm 0.35$ & $3.87 \pm 0.45$ & $3.97 \pm 0.30$ & $4.22 \pm 0.28$ \\
\hline IZL (mm) & $89.1 \pm 3.5$ & $94.2 \pm 6.1$ & $95.2 \pm 5.9$ & $98.0 \pm 5.7$ & $91.9 \pm 5.6$ & $95.2 \pm 2.2$ & $96.9 \pm 1.4$ \\
\hline $\mathrm{GP}(\mathrm{mm})$ & $11.2 \pm 2.3$ & $12.9 \pm 2.1$ & $13.7 \pm 1.8$ & $14.2 \pm 1.6$ & $13.9 \pm 1.9$ & $14.4 \pm 3.0$ & $14.4 \pm 3.3$ \\
\hline ONSD (mm) & $3.67 \pm 0.46$ & $3.84 \pm 0.49$ & $4.12 \pm 0.43$ & $4.25 \pm 0.46$ & $4.20 \pm 0.29$ & $3.47 \pm 0.34$ & $3.75 \pm 0.43$ \\
\hline LD (HU) & $128.26 \pm 12.30$ & $134.24 \pm 17.17$ & $138.53 \pm 15.44$ & $147.42 \pm 15.68$ & $145.36 \pm 13.14$ & $148.26 \pm 11.46$ & $156.67 \pm 6.80$ \\
\hline
\end{tabular}

* Orbital volume $\left(\mathrm{cm}^{3}\right)$; Ocular diameter $(\mathrm{mm})$; Medial rectus $(\mathrm{mm})$; Lateral rectus (mm); Superior Group $(\mathrm{mm})$; Inferior rectus ( $\mathrm{mm})$; Interzygomatic line (mm); Globe position ( $\mathrm{mm})$; Optic nerve sheath diameter $(\mathrm{mm})$; Lens density $(\mathrm{HU})$

\section{DISCUSSION}

Although $\mathrm{MRI}$ and USG are preferred imaging modalities for orbit and periorbital tissues, CT offers rapid image acquisition, less operator dependent as compared to USG and is easily available than MRI. ${ }^{11}$ Although the ionizing radiation effect limits it routine utilization, CT may be considered essential when other means of accurate measurement of ocular dimension is contraindicated or unemployable. We kept our study limited to the patients undergoing PNS CT examination and didn't consider carrying out the study in the patients undergoing cranial CT examinations because that would result in increasing the scan field of view and thus increasing unnecessary radiation dose to the patients .

Tracing the computer image is likely to cause an error. The error is shown to be $1.6 \%$ by McGurk et al. The accuracy of measurement was not investigated, because the error is considered to be very small and it was difficult to measure the same orbital area twice as the traced area in this study. ${ }^{12}$ The accuracy of measurement was not studied in the present study.

The volume of the eye ball changes during life time as observed in this study. Our result showed the increase in ocular diameter, a major component of volume of eye ball, with age in concordance to the study on 1232 adult Chinese in Singapore which showed the axial length i.e. ocular diameter (a major variable in the calculation of ocular volume) increased until 50 years before reduction was observed. ${ }^{13}$ However, we didn't find the significant reduction in the ocular diameter after 50 years of age. The reason could be inadequate sample of subjects after 50 years of age enrolled in our study as compared to that below 50 years.

The mean orbital volume in our study is slightly larger than the one measured in Japanese population and the mean orbital volume in our study was significantly larger in men than the female in accordance to the finding in Japanese population. ${ }^{14}$ It appears that the values they recorded differed either because of the different sample populations, varied population ages and habitus or from racial differences. The higher orbital volume in male may result from human sexual dimorphism (male body habitus is generally bigger than that of the females). ${ }^{15}$

Several studies have been carried out in the past which evaluated diameters of extraocular muscles with varying window settings. Every change in the window level and width settings results in different values with respect to the muscle size which concludes that the window settings must be uniform for the precise measurements of the muscle sizes. ${ }^{3}$ Thus, normative data of this study would be valid for specific window level and width settings with window width and window level 300 and 50 respectively.

In the present study, the mean diameter of the rectus muscles were $3.67 \mathrm{~mm}, 3.36 \mathrm{~mm}, 3.87 \mathrm{~mm}$ and $3.74 \mathrm{~mm}$ in medial, lateral, superior complex and inferior rectus muscles respectively. These findings were in concordance to studies done by Jong Soo Lee16 and Sukalya. ${ }^{17}$ Most of the studies showed similar ranking of thickness of extraocular muscles, inferior $>$ superior $>$ medial $>$ lateral. Our study also showed similar ranking except for higher thickness of superior complex in our study as we included superior levator palpebrae in the measurement. The present study revealed that the mean diameters of the extraocular muscles were little larger in males than females but not statistically significant. This may be due to a larger orbit size in accordance to larger body habitus in male.

The mean ONSD found in this study was lower than the ONSD measured in Korean populations 
(4.20 mm, ranging from 3.6 to $4.8 \mathrm{~mm}) .{ }^{16}$ This difference in measurements may be due to racial inheritance, and possibly also by environmental influence. Altogether, these results imply that the optic nerve sheath measurements differ from one population to another, even among neighboring Asian countries, thus highlighting the need to establish normative values which are specific for the Nepalese population.

The LD measurement showed increasing value as the age progresses. The mean attenuation value was found to be higher in females than in males but they are not statistically significant $(p>0.05)$. This study provided us with the mean as well as the range of attenuation value of the lens. It is predictable that changes in lens thickness and attenuation would also be noted in cases of cataracts on $\mathrm{CT}^{9}$ but further studies are required to confirm this.

\section{CONCLUSION}

Sizes of orbital structures observed in this study population are significantly smaller than reference values available in literature from European, African and Korean populations. This study will be helpful to radiologists and ophthalmologists to accurately assess various orbital structures with a practical quantitative method.

\section{CONFLICT OF INTEREST}

None declared.

\section{REFERENCES}

1. Igbinedion BO, Ogbeide OU. Measurement of normal ocular volume by the use of computed tomography. 2013;16(3):315-9.

2. Trokel SL HS. Recognition and differential diagnosis of enlarged extraocular muscles in computed tomographytle. Am I Ophthalmol. 1979;87:503-12.

3. Rothfus WE CH. Extraocular muscle enlargement: a CT review.
Radiology. 1984;151:677-81.

4. Patrinely JR, Osborn AG, Anderson RL, et al. Computed tomographic features of nonthyroid extraocular muscle enlargement. OphthsImology. 1989;96:1038-47.

5. Kimberly HH, Shah S, Marill K N V. Correlation of optic nerve sheath diameter with direct measurement of intracranial pressure. Acad Emerg Med. 2008;15(2):201-4.

6. Geeraerts T, Launey Y, Martin L, et al. Ultrasonography of the optic nerve sheath may be useful for detecting raised intracranial pressure after severe brain injury. Intensive Care Med. 2007;33(10):170411.

7. Acer N, Sahin B, Ergur $H$, et al. Stereological estimation of the orbital volume: a criterion standard study. I Craniofac Surg. 20(3):921-5.

8. Ahmadi H, Shams PN, Davies NP, et al. Age-related changes in the normal sagittal relationship between globe and orbit. J Plas Reconstr Aesthet Surg. 60(3):246-50.

9. Boorstein M, Wong KT, Grossmani RI. CT Diagnosis of Unsuspected Traumatic Cataracts in Patients with Complicated Eye Injuries: Significance of Attenuation Value of the Lens.

10. Vaiman M, Abuita R, Bekerman I. Optic nerve sheath diameters in healthy adults measured by computer tomography. Int I Ophthalmol [Internet]. 2015;8(6):1240—1244.

11. Hande PC, Talwar I. Multimodality imaging of the orbit. Indian J Radiol Imaging [Internet]. 2012 Jul;22(3):227-39.

12. McGurk M, Whitehouse RW, Taylor PM, et al. Orbital volume measured by a low-dose CT scanning technique. Dentomaxillofacial Radiol [Internet]. 1992 May 1;21(2):70-2.

13. Wong TY, Foster PJ, Ng TP, et al. Variations in Ocular Biometry in an Adult Chinese Population in Singapore: The Tanjong Pagar Survey. Invest Ophthalmol Vis Sci. 2001 Jan 1;42(1):73-80.

14. Furuta M. Measurement of Orbital Volume by Computed Tomography: Especially on the Growth of the Orbit. Jpn J Ophthalmol. 2001 Nov-Dec;45(6):600-6.

15. Nieves JW, Formica C, Ruffing J, et al. Males Have Larger Skeletal Size and Bone Mass Than Females, Despite Comparable Body Size. J Bone Miner Res [Internet]. 2005 Mar 1;20(3):529-35.

16. Lee JS, Lim DW, Lee SH, et al. Normative measurements of Korean orbital structures revealed by computerized tomography. 1998;197-200.

17. Lerdlum S, Boonsirikamchai P, Setsakol E. Normal Measurements of Extraocular Muscle Using Computed Tomography. J Med Assoc Thai. 2007 Feb 1;90:307-12. 